www.jmscr.igmpublication.org

Impact Factor 5.84

Index Copernicus Value: 83.27

ISSN (e)-2347-176x ISSN (p) 2455-0450

crossref DOI: _https://dx.doi.org/10.18535/jmscr/v5i3.188

Journal Of Medical Science And Clinical Research

IGM Publication

An Official Publication of IGM Publication

\title{
Comparison of Vacuum Assisted Closure with Conventional Dressing
}

\author{
Authors \\ Dr I J Jinu, Dr Lyke Xavier, Dr D Dimmy Harold \\ TD Medical College, Alappuzha
}

\section{INTRODUCTION}

One of the most common causes for admission in surgical ward is non healing ulcer. In which diabetes is the most common etiology. In most of the cases, hospital stay of many weeks is required for management of the above. In many cases they ultimately go for amputation. Acute and chronic wounds affect at least $1 \%$ of the population. Regardless of etiology, wounds are difficult to treat if coexisting factors (eg, infection or diabetes mellitus) prevent regular wound healing.

Wounds represent a significant risk factor for hospitalization, amputation, sepsis, and even death, and from the patient's perspective, wound therapy is often un-comfortable or painful. In all sense patients turns to be a burden for society and family.

Vacuum assisted closure is a universally accepted method for dressing. It has proved its efficacy for wound dressing. Faster wound healing, shorter hospital stay.

Still in our hospital, majority of dressings are conventional. My aim is to show the advantage of V.A.C over conventional dressing in our hospital

Key words: vaccum assisted closure; wound healing; non healing ulcer

MeSH terms: vaccum assisted closure; non healing ulcer

\section{ETHICAL CONSIDERATIONS}

Study has been conducted after getting approval from institutional ethical committee. A written informed consent has been taken from all the patients included in the study. Patients participating in the study did not have to incur any expenses. The anonymity of each individual has been maintained.

\section{METHODOLOGY}

\section{STUDY DESIGN}

Case control study

\section{STUDY SETTING}

Study is conducted at Govt medical collage Alappuzha, which is tertiary Centre. Patients are selected from general surgery wards

\section{PERIOD OF STUDY}

Study is conducted from April 1 to Nov. 30, 2016, total 8 months

\section{SAMPLE SIZEANDMETHOD OF} ALLOCATION OF GROUP

Cases are selected from the surgical ward (2 specific wards allocated) from April 1 to November 30

Controls selected from the during this period from another surgical ward.

Total 30 cases and 30 controls; they were selected randomized by the admission. As my study period was from april 1 to Nov 30, ie total of 8 months. 
Patients who agreed and gave consent for VAC from surgical ward 15 were 30 . So 30 patients were selected from ward 12.

\section{INTERVENTION}

Patients included in study are classified according to grade of ulcer. All grades are included except grade 5. Other patients excluded are patients with

- Gangrenous foot

- Suspicious of anaerobic infections

- Exposed blood vessels

- Active bleeding

- Undebrided wound

- Malignancy

After debridement of wound V.A.C dressing is applied, after bleeding gets stopped. Pre V.A.C \& post V.A.C C\&S is taken. Dressing is given for 5 days. doppler study, $\mathrm{x}$ ray taken.

CONTROL group with conventional saline soaked dressing

\section{OUTCOME VARIABLES}

- rate of outcome,

- hospital stay,

- pus C\&S before \& after V.A.C

\section{METHOD OF STUDY}

During the period of study, I randomized the patients to CASES and CONTROL as surgical ward 15,23 Apr. 1 to nov. 30

Controls selected from the surgical ward 12 during this period.

After debridement of wound V.A.C dressing is applied, after bleeding gets stopped. Pre V.A.C \& post V.A.C C\&S is taken. Dressing is given for 5 days. doppler study, $\mathrm{x}$ ray taken.

CONTROL group with conventional dressing

Status of the patient at the time of discharge is noted

\section{MATERIALS USED FOR STUDY}

- Performa

- Camera cover

- Transparent adhesive plaster

- Sponge

- Suction drain/ suction apparutus available

\section{Data analysis}

\section{Statistical method}

\section{Chisquare test}

All the analysis was done using qualitative variables in statistical package for social sciences (SPSS) version 16.

\section{RESULTS}

Table 1 Hospital Stay

\begin{tabular}{|ll|l|l|c|c|}
\hline \multicolumn{1}{|c|}{$\begin{array}{l}\text { case/cont } \\
\text { rol }\end{array}$} & $\mathrm{N}$ & Mean & $\begin{array}{c}\text { Std. } \\
\text { Deviation }\end{array}$ & $\begin{array}{c}\text { Std. Error } \\
\text { Mean }\end{array}$ \\
\hline STAY & Case & 30 & 21.17 & 7.940 & 1.450 \\
& control & 30 & 28.53 & 7.045 & 1.286 \\
\hline
\end{tabular}

Mean hospital stay in cases is 21 compared to stay of 28 in control group.

Table 2 PREVAC * POSTVAC $\mathrm{C} \& \mathrm{~S}$ Crosstabulation

\begin{tabular}{|lc|c|c|c|}
\hline \multirow{2}{*}{} & & \multicolumn{2}{|c|}{ POSTVAC } & \multirow{2}{*}{} \\
\cline { 2 - 4 } & & sterile & non sterile & \multirow{2}{*}{ Total } \\
\hline PREVAC & sterile & 9 & 0 & 9 \\
& non sterile & 19 & 2 & 21 \\
Total & & 28 & 2 & 30 \\
\hline
\end{tabular}

Patients with sterile pre V.A.C C\&S is not turning non sterile after V.A.C, but $90 \%$ non-sterile turns sterile after V.A.C

Table 3 Xray * PLAN Crosstabulation

Count

\begin{tabular}{|lc|c|c|c|l|}
\hline \multirow{2}{*}{} & & \multicolumn{3}{|c|}{ PLAN } & \multirow{3}{*}{} \\
\cline { 3 - 5 } & & & \multicolumn{2}{|c|}{} & amputat \\
ion & Total \\
\hline Xray & OM present & 5 & 9 & 2 & 16 \\
& OM absent & 7 & 7 & 0 & 14 \\
Total & & 12 & 16 & 2 & 30 \\
\hline
\end{tabular}

There were no much difference observed in outcome of the patient with or without osteomyelitis on applying V.A.C.

Table 4 DOPPLER * PLAN Cross tabulation

Count

\begin{tabular}{|c|c|c|c|c|c|}
\hline & \multicolumn{3}{|c|}{ PLAN } & \multirow[t]{2}{*}{ Total } \\
\hline & & $\begin{array}{c}\text { Disch } \\
\text { arge }\end{array}$ & SSG & $\begin{array}{l}\text { amput } \\
\text { ation }\end{array}$ & \\
\hline \multirow{4}{*}{\multicolumn{2}{|c|}{ 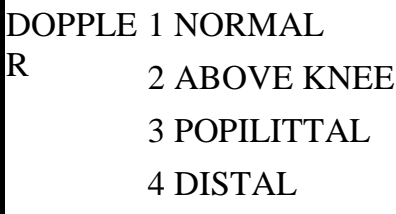 }} & 9 & 15 & 0 & 24 \\
\hline & & 1 & 0 & 1 & 2 \\
\hline & & 0 & 0 & 1 & 1 \\
\hline & & 2 & 1 & 0 & 3 \\
\hline \multicolumn{2}{|c|}{ Total } & 12 & 16 & 2 & 30 \\
\hline
\end{tabular}

SSG as outcome is more in patients with normal Doppler study . 15 out of 16 patients undergone SSG is having normal Doppler study. 
Table 5 case/control * plan at end of $\mathrm{Rx}$

Cross tabulation

\begin{tabular}{|ll|c|c|c|c|}
\hline & & $\begin{array}{c}\text { DISCH } \\
\text { ARGE }\end{array}$ & SSG & $\begin{array}{c}\text { AMPUTATI } \\
\text { ON }\end{array}$ & \\
\hline case/control & case & 12 & 16 & 2 & 30 \\
& control & 22 & 0 & 8 & 30 \\
Total & & 34 & 16 & 10 & 60 \\
\hline
\end{tabular}

Patients with V.A.C dressing have more split skin graft before discharge, less rate of amputation rate

\section{DISCUSSION}

This study was conducted on a group of 60 patients. They were divided into case and control. It was single blind as case were selected as admitted to specific ward during the period of study.

It was found that outcome of the patient after V.A.C were better than conventional dressing

\section{Figure: 1}

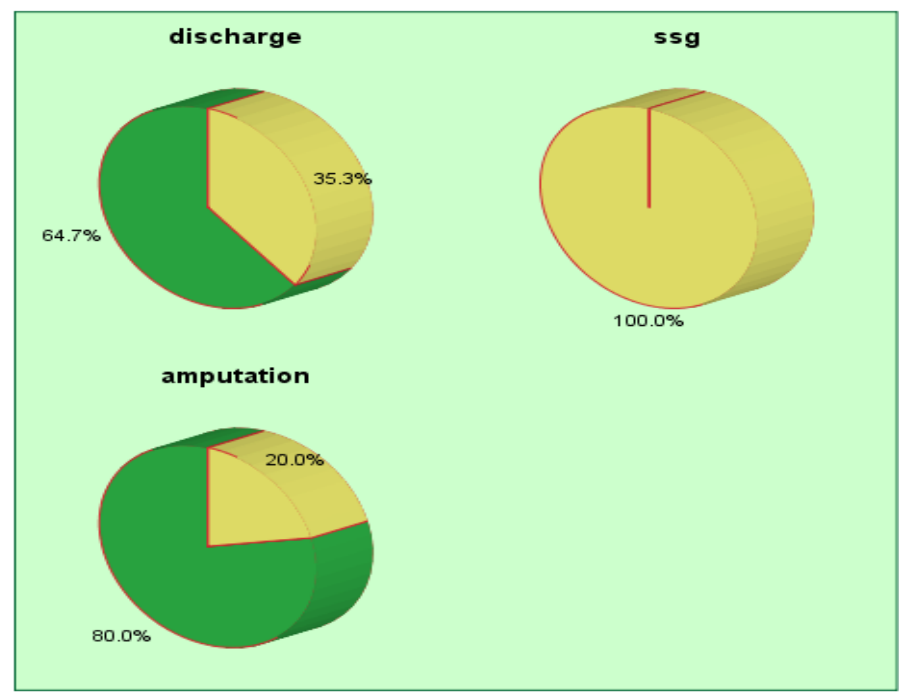

Table 6

\begin{tabular}{|ll|c|c|c|c|}
\hline \multirow{2}{*}{} & & \multicolumn{3}{|c|}{ plan at end of Rx } & \multirow{3}{*}{} \\
\cline { 3 - 5 } & & $\begin{array}{c}\text { DISCH } \\
\text { ARGE }\end{array}$ & SSG & $\begin{array}{c}\text { AMPUTA } \\
\text { TION }\end{array}$ & Total \\
\hline case/control & case & 12 & 16 & 2 & 30 \\
& control & 22 & 0 & 8 & 30 \\
Total & 34 & 16 & 10 & 60 \\
\hline
\end{tabular}

Table : 7 Chi-Square Tests

\begin{tabular}{|l|c|c|c|}
\hline & Value & df & $\begin{array}{c}\text { Asymp. Sig. (2- } \\
\text { sided) }\end{array}$ \\
\hline Pearson Chi-Square & $22.541^{\mathrm{a}}$ & 2 & .001 \\
Likelihood Ratio & 29.021 & 2 & .001 \\
N of Valid Cases & 60 & & \\
\hline
\end{tabular}

Chi-square test shows study is significant ( $\mathrm{p}$ value of .001).

$$
\begin{aligned}
& \text { case/control } \\
& \square \text { case } \\
& \text { control }
\end{aligned}
$$

HOSPITAL STAY

\begin{tabular}{|c|c|c|c|c|c|c|c|c|}
\hline & & \multicolumn{7}{|c|}{ t-test for Equality of Means } \\
\hline & & \multirow[b]{2}{*}{$\mathrm{t}$} & \multirow[b]{2}{*}{ df } & \multirow[b]{2}{*}{$\begin{array}{l}\text { Sig. (2- } \\
\text { tailed) }\end{array}$} & \multirow{2}{*}{$\begin{array}{l}\text { Mean } \\
\text { Differe } \\
\text { nce }\end{array}$} & \multirow{2}{*}{$\begin{array}{c}\text { Std. } \\
\text { Error } \\
\text { Differen } \\
\text { ce }\end{array}$} & \multicolumn{2}{|c|}{$\begin{array}{l}\text { 95\% Confidence } \\
\text { Interval of the } \\
\text { Difference }\end{array}$} \\
\hline & & & & & & & Lower & Upper \\
\hline \multirow[t]{2}{*}{ STAY } & Equal variances assumed & -3.801 & 58 & .000 & -7.367 & 1.938 & -11.246 & -3.487 \\
\hline & Equal variances not assumed & -3.801 & 57.191 & .000 & -7.367 & 1.938 & -11.247 & -3.486 \\
\hline
\end{tabular}

Table : 8 
Table : 9

Group Statistics

\begin{tabular}{|ll|c|c|c|c|}
\hline & case/control & $\mathrm{N}$ & Mean & Std. Deviation & Std. Error Mean \\
\hline STAY & Case & 30 & 21.17 & 7.940 & 1.450 \\
& & & & & \\
& control & 30 & 28.53 & 7.045 & 1.286 \\
\hline
\end{tabular}

Chi-square test shows study is significant (p value of .001, -3.801)

Table : 10 PREVAC * POSTVAC C\&S Cross tabulation

\begin{tabular}{|ll|l|l|l|}
\hline \multirow{2}{*}{} & \multicolumn{2}{|l|}{ POSTVAC } & \multicolumn{1}{l|}{} \\
\cline { 3 - 4 } & & sterile & non sterile & Total \\
\hline PREVAC & sterile & 9 & 0 & 9 \\
& non sterile & 19 & 2 & 21 \\
Total & & 28 & 2 & 30 \\
\hline
\end{tabular}

V.A.C dressing turns non sterile wound to sterile.Applying V.A.C. doesn't turns sterile wound to non-sterile

Table : 11 Chi-Square Tests

\begin{tabular}{|l|c|c|c|c|c|}
\hline & Value & df & $\begin{array}{c}\text { Asymp. Sig. (2- } \\
\text { sided) }\end{array}$ & $\begin{array}{c}\text { Exact Sig. (2- } \\
\text { sided) }\end{array}$ & $\begin{array}{c}\text { Exact Sig. (1- } \\
\text { sided) }\end{array}$ \\
\hline Pearson Chi-Square & $.918^{\mathrm{a}}$ & 1 & .002 & & \\
Continuity Correction ${ }^{\mathrm{b}}$ & .026 & 1 & .873 & & \\
Likelihood Ratio & 1.487 & 1 & .223 & & \\
Fisher's Exact Test & & & & & \\
Linear-by-Linear Association & .888 & 1 & .346 & & \\
N of Valid Cases & 30 & & & & \\
\hline
\end{tabular}

Chi-square test shows study is significant ( $\mathrm{p}$ value .002)

Table : 12 OSTEOMYLITIS * OUTCOME Crosstabulation

\begin{tabular}{|cc|c|c|c|c|}
\hline & \multicolumn{3}{|c|}{ PLAN } & \\
\cline { 3 - 5 } & & DISCHARGE & SSG & AMPUTATION & Total \\
\hline Xray & OM present & 5 & 9 & 2 & 16 \\
& OM absent & 7 & 7 & 0 & 14 \\
Total & 12 & 16 & 2 & 30 \\
\hline
\end{tabular}

Table : 13 Chi-Square Test

\begin{tabular}{|l|c|c|c|}
\hline & Value & df & Asymp. Sig. (2-sided) \\
\hline Pearson Chi-Square & $2.461^{\mathrm{a}}$ & 2 & .292 \\
Likelihood Ratio & 3.225 & 2 & .199 \\
Linear-by-Linear & 1.982 & 1 & .159 \\
Association & & & \\
N of Valid Cases & 30 & & \\
\hline
\end{tabular}

Chi-Square Test shows study is not significant as p-value is .292 that means OSTEOMYLITIS is not a contraindication for V.A.C dressing. 
Table : 14 DOPPLER * PLAN Crosstabulation

\begin{tabular}{|ll|c|c|c|c|}
\hline & & \multicolumn{3}{|c|}{ PLAN } & \multirow{2}{*}{ Total } \\
\cline { 2 - 5 } & & DISCHARGE & SSG & AMPUTATION & TA \\
\hline DOPPLER & 1 & 9 & 15 & 0 & 24 \\
& 2 & 1 & 0 & 1 & 2 \\
& 3 & 0 & 0 & 1 & 1 \\
& 4 & 2 & 1 & 0 & 3 \\
Total & 12 & 16 & 2 & 30 \\
\hline
\end{tabular}

Patients with normal Doppler shows better outcome. Almost 100\% patients undergone SSG were having normal Doppler.

TABLE : 15

Chi-Square Tests

\begin{tabular}{|l|c|c|c|}
\hline & Value & $\mathrm{df}$ & Asymp. Sig. (2-sided) \\
\hline Pearson Chi-Square & $23.724^{\mathrm{a}}$ & 6 & .001 \\
Likelihood Ratio & 14.592 & 6 & .024 \\
Linear-by-Linear Association & .011 & 1 & .916 \\
N of Valid Cases & 30 & & \\
\hline
\end{tabular}

Chi-Square Test shows study is significant as p-value is .OO1

\section{CONCLUSION}

V.A.C dressing decreases Hospital stay

V.A.C dressing improves pus culture sensitivity

V.A.C dressing improves outcome, more SSG

V.A.C dressing has better result in patients with Normal Doppler

V.A.C dressing has good result in patients with non-active osteomyelitis

\section{BIBLIOGRAPHY}

1. Whitworth I. History and development of negative pressure therapy. In: Banwell PE, Teot L, editors. 1st International Topical Negative Pressure Focus Group Meeting. Faringdon, UK: TPX Communications; 2004. p. 22-6.

2. Fleischmann W, Strecker W, Bombelli M, et al. [Vacuum sealing as treatment of soft tissue dam-age in open fractures]. Unfallchirurg 1993; 96(9):488-92 (in German).

3. Argenta LC, Morykwas MJ. Vacuumassisted closure: a new method for wound control and treatment: clinical experience. Ann Plast Surg 1997; 38(6):563-76 [discussion: 577].
4. Morykwas MJ, Argenta LC, SheltonBrown EI,et al. Vacuum-assisted closure: a new methodfor wound control and treatment: animal studies and basic foundation. Ann Plast Surg 1997; 38(6):553-62.

5. Skagen K, Henrikson O. Changes in subcutaneous blood flow during locally applied negativepressure to the skin. ActaPhysiolScand 1983; 117(3):411-4.

6. Fentem PH, Matthews JA. The duration of the increase in arterial inflow during the exposure ofthe forearm to subatmospheric pressure. J Physiol 1970; 210(2):65-6.

7. Banwell PE, Morykwas MJ, Jennings DA, etal.Dermalmicrovascular flow in experimental partial thickness burns: the effect of topical subatmospheric pressure. J Burn Care Rehabil2000; 21:s161.

8. Fabian TS, Kaufman HJ, Lett ED, et al. The evaluation of subatmospheric pressure and hyper-baric oxygen in ischemic fullthickness woundhealing. Am Surg 2000; 66(12):1136-43.

9. Fleischmann W, Becker U, Bischoff M, etal.Vacuum sealing: indication technique 
and re-sults. Eur J Orthop Surg Trauma 1995; 5:37-40.

10. Weed T, Ratliff C, Drake DB. Quantifying bacterial bioburden during negative pressure wound therapy: does the wound VAC enhance bacterial clearance? Ann Plast Surg 2004; 52(3):276-9[discussion: 279-80]. 